\title{
Solving Singularly Perturbed Multipantograph Delay Equations Based on the Reproducing Kernel Method
}

\author{
F. Z. Geng and S. P. Qian \\ Department of Mathematics, Changshu Institute of Technology, Changshu, Jiangsu 215500, China \\ Correspondence should be addressed to F. Z. Geng; gengfazhan@sina.com
}

Received 13 November 2013; Accepted 20 January 2014; Published 27 February 2014

Academic Editor: Valery Y. Glizer

Copyright ( 2014 F. Z. Geng and S. P. Qian. This is an open access article distributed under the Creative Commons Attribution License, which permits unrestricted use, distribution, and reproduction in any medium, provided the original work is properly cited.

A numerical method is presented for solving the singularly perturbed multipantograph delay equations with a boundary layer at one end point. The original problem is reduced to boundary layer and regular domain problems. The regular domain problem is solved by combining the asymptotic expansion and the reproducing kernel method (RKM). The boundary layer problem is treated by the method of scaling and the RKM. Two numerical examples are provided to illustrate the effectiveness of the present method. The results from the numerical example show that the present method can provide very accurate analytical approximate solutions.

\section{Introduction}

In this paper, we consider the following singularly perturbed multipantograph delay equation:

$$
\begin{gathered}
\varepsilon u^{\prime \prime}(x)+a(x) u^{\prime}(x)+\sum_{i=1}^{m} b_{i}(x) u\left(q_{i} x\right)=f(x), \\
0<x<1, \\
u(0)=\alpha, \quad u(1)=\gamma,
\end{gathered}
$$

where $0<\varepsilon \ll 1,0<q_{i} \leq 1, m$ is a positive integer and $a(x), b_{i}(x)$ and $f(x)$ are assumed to be sufficiently smooth, such that (1) has a unique solution with a boundary layer at $x=0$.

Singularly perturbed problems arise frequently in applications including geophysical fluid dynamics, oceanic and atmospheric circulation, chemical reactions, and optimal control. These problems are characterized by the presence of a small parameter that multiplies the highest order derivative, and they are stiff and there exist boundary layers where the solutions change rapidly.

Functional differential equations with proportional delays are usually referred to as pantograph equations. These equations arise in a variety of applications, such as number theory, electrodynamics, astrophysics, nonlinear dynamical systems, probability theory on algebraic structure, quantum mechanics, and cell growth.

Recently, singularly perturbed delayed differential equations have attracted significant attention. The numerical treatment of such problems presents some major computational difficulties, and therefore discussion on numerical solutions of singularly perturbed delayed differential equation is rare. Amiraliyev et al. $[1,2]$ proposed a uniform numerical method for dealing with singularly perturbed delay initial value problems. Kadalbajoo et al. [3-6] presented some effective methods for solving singularly perturbed delay boundary value problems. In [7], Amiraliyev and Cimen also introduced a numerical method for singularly perturbed delay boundary value problems. In [8], Rai and Sharma described a numerical method based on fitted operator finite difference scheme for the boundary value problems for singularly perturbed delay differential equations with turning point and mixed shifts.

Reproducing kernel theory has important applications in numerical analysis, differential equations, probability, and statistics, amongst other fields [9-20]. Recently, using the reproducing kernel method (RKM), the authors have discussed various differential equations [11-20]. However, it is very difficult to expand the application of the RKM to singularly perturbed delayed differential equations. Geng [13] 
developed a method for solving a class of singularly perturbed boundary value problems based on the RKM and a proper transformation. Nevertheless, this method fails to solve singularly perturbed delayed differential equations.

In this paper, based on the RKM presented in $[9,11]$, an effective numerical method will be presented for solving singularly perturbed delayed boundary value problem (1).

The rest of the paper is organized as follows. In the next section, the numerical technique for (1) is introduced. Error analysis is introduced in Section 2. The numerical example is given in Section 3. Section 4 ends this paper with a brief conclusion.

\section{Numerical Method}

We divide the domain $[0,1]$ into two subdomains, namely, $[0, K \varepsilon]$ and $[K \varepsilon, 1]$, where $K$ is a positive real number. The asymptotic approximation technique and the RKM are combined to solve (1) in the regular domain $[K \varepsilon, 1]$. And then the value of asymptotic approximation in the regular domain is used as the boundary condition at the so-called transition point $x=K \varepsilon$. In the boundary layer domain $[0, K \varepsilon],(1)$ is solved by combining the method of scaling and the RKM. After solving both the regular and boundary layer domain problems their solutions are combined to obtain an approximate solution to the original problem over the entire domain $[0,1]$.

2.1. Solution of the Regular Domain. We seek the regular region solution as an asymptotic expansion of the form

$$
u_{r}(x)=\sum_{k=0}^{M} \varepsilon^{k} u_{k}(x)
$$

where $u_{k}(x)$ are unknown functions to be determined.

Substituting $u_{r}(x)$ into (1) and equating the coefficients of like powers of $\varepsilon$, we obtain

$$
\begin{array}{cc}
a(x) u_{0}^{\prime}(x)+\sum_{i=1}^{m} b_{i}(x) u_{0}\left(q_{i} x\right)=f(x), & u_{0}(1)=\gamma, \\
a(x) u_{1}^{\prime}(x)+\sum_{i=1}^{m} b_{i}(x) u_{1}\left(q_{i} x\right)=-u_{0}^{\prime \prime}(x), & u_{1}(1)=0, \\
a(x) u_{2}^{\prime}(x)+\sum_{i=1}^{m} b_{i}(x) u_{2}\left(q_{i} x\right)=-u_{1}^{\prime \prime}(x), & u_{2}(1)=0,
\end{array}
$$$$
a(x) u_{M}^{\prime}(x)+\sum_{i=1}^{m} b_{i}(x) u_{M}\left(q_{i} x\right)=-u_{M-1}^{\prime \prime}(x), \quad u_{M}(1)=0
$$

By the RKM, the solutions of the above equations $u_{0}, u_{1}, u_{2}, \ldots, u_{M}(x)$ can be approximated by

$$
\begin{gathered}
u_{0, N}(x)=\gamma+\sum_{i=1}^{N} \sum_{k=1}^{i} \beta_{i k} f_{0}\left(x_{k}\right) \bar{\psi}_{i}(x), \\
u_{1, N}(x)=\sum_{i=1}^{N} \sum_{k=1}^{i} \beta_{i k} f_{1}\left(x_{k}\right) \bar{\psi}_{i}(x), \\
u_{2, N}(x)=\sum_{i=1}^{N} \sum_{k=1}^{i} \beta_{i k} f_{2}\left(x_{k}\right) \bar{\psi}_{i}(x),
\end{gathered}
$$

$$
u_{M, N}(x)=\sum_{i=1}^{N} \sum_{k=1}^{i} \beta_{i k} f_{M}\left(x_{k}\right) \bar{\psi}_{i}(x)
$$

where $f_{0}(x)=f(x)-\gamma \sum_{i=1}^{m} b_{i}(x), f_{1}(x)=-u_{0, N}^{\prime \prime}(x), f_{2}(x)=$ $-u_{1, N}^{\prime \prime}(x), \ldots, f_{M}(x)=-u_{M-1, N}^{\prime \prime}(x)$.

Therefore, the solution of regular region $u_{r}(x)$ can be approximated by

$$
u_{r, N}=\sum_{k=0}^{M} \varepsilon^{k} u_{k, N}(x)
$$

In the following, we will show how to solve (3) using the RKM in detail.

Consider the following operator equation:

$$
\begin{gathered}
L v(x) \equiv a(x) v^{\prime}(x)+\sum_{i=1}^{m} b_{i}(x) v\left(q_{i} x\right)=h(x), \quad 0<x<1, \\
v(1)=0 .
\end{gathered}
$$

Under the assumption that (3) has a unique solution, we will give the approximate solution of (3) in the reproducing kernel space $W_{2}^{2}[0,1]$.

The reproducing kernel space $W_{2}^{2}[0,1]$ is defined as $W_{2}^{2}[0,1]=\left\{u(x) \mid u(x), u^{\prime}(x)\right.$ are absolutely continuous real valued functions, $\left.u^{\prime \prime}(x) \in L^{2}[0,1], u(1)=0\right\}$. The inner product and norm in $W_{2}^{2}[0,1]$ are given, respectively, by

$$
\begin{gathered}
(u(y), v(y))_{W_{2}^{2}}=u(0) v(0)+u(1) v(1)+\int_{0}^{1} u^{\prime \prime} v^{\prime \prime} d y, \\
\|u\|_{W_{2}^{2}}=\sqrt{(u, u)_{W_{2}^{2}}}, \quad u, v \in W_{2}^{2}[0,1] .
\end{gathered}
$$

Its reproducing kernel is

$$
R_{x}(y)= \begin{cases}\frac{1}{6}(x-1)\left(y^{3}+\left(x^{2}-2 x+6\right) y-6\right), & y \leq x, \\ \frac{1}{6}(y-1)\left(x^{3}+\left(y^{2}-2 y+6\right) x-6\right), & y>x .\end{cases}
$$




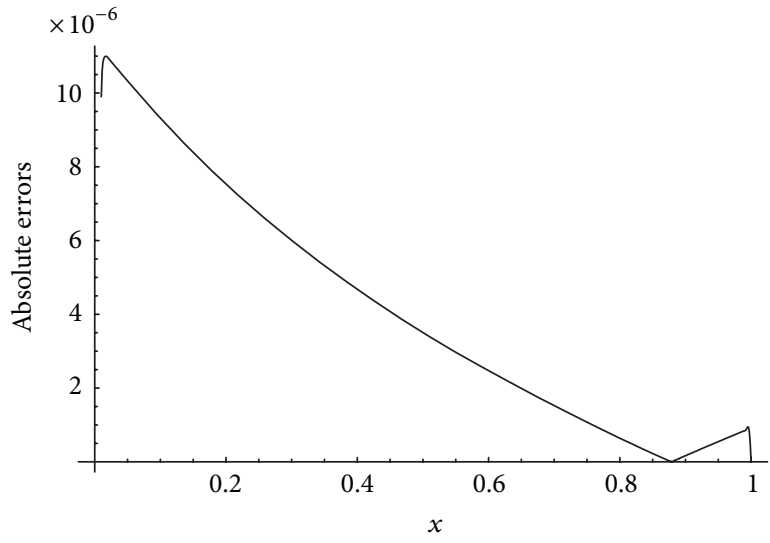

(a)

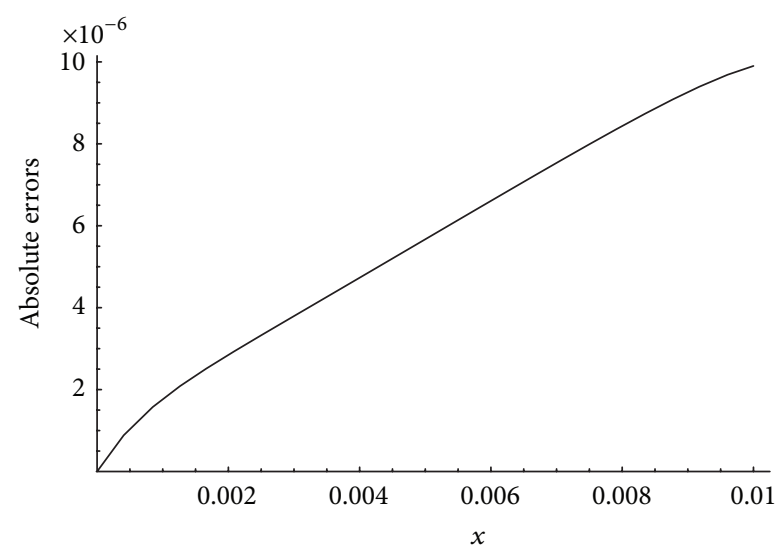

(b)

FIGURE 1: Absolute errors $\left|u(x)-u_{200}(x)\right|$ of Example 1 for $\varepsilon=10^{-3}$ ((a): regular domain; (b): boundary later domain).

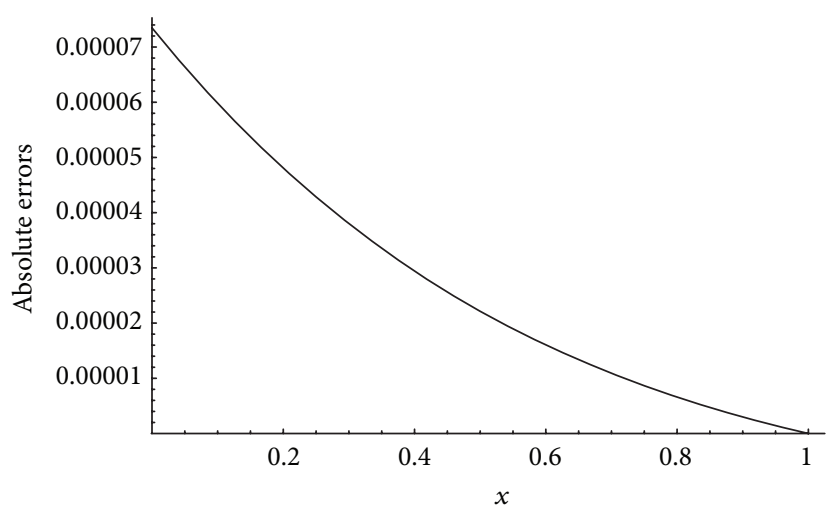

(a)

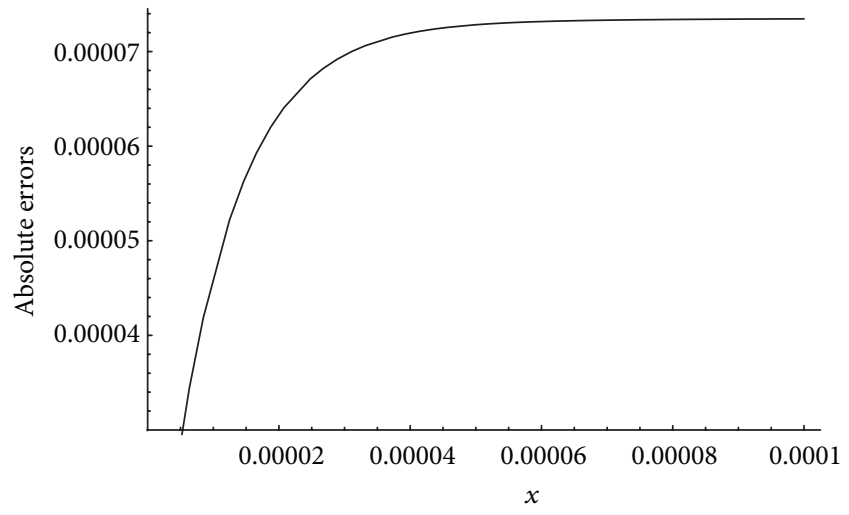

(b)

FIGURE 2: Absolute errors $\left|u(x)-u_{200}(x)\right|$ of Example 1 for $\varepsilon=10^{-5}$ ((a): regular domain; (b): boundary later domain).

For detailed method of obtaining reproducing kernel $R_{x}(y)$, please refer to $[9,15]$.

In (6), it is clear that $L: W_{2}^{2}[0,1] \rightarrow W_{2}^{1}[0,1]$ is a bounded linear operator (please see [9] for the definition of $\left.W_{2}^{1}[0,1]\right)$. Put $\varphi_{i}(x)=\bar{R}_{x_{i}}(x)$ and $\psi_{i}(x)=L^{*} \varphi_{i}(x)$, where $\bar{R}_{x}(y)$ is the reproducing kernel of $W_{2}^{1}[0,1], L^{*}$ is the adjoint operator of $L$. The orthonormal system $\left\{\bar{\psi}_{i}(x)\right\}_{i=1}^{\infty}$ of $W_{2}^{2}[0,1]$ can be derived from the Gram-Schmidt orthogonalization process applied to $\left\{\psi_{i}(x)\right\}_{i=1}^{\infty}$,

$$
\bar{\psi}_{i}(x)=\sum_{k=1}^{i} \beta_{i k} \psi_{k}(x), \quad\left(\beta_{i i}>0, i=1,2, \ldots\right) .
$$

Theorem 1. For (6), if $\left\{x_{i}\right\}_{i=1}^{\infty}$ is dense on $[0,1]$, then $\left\{\psi_{i}(x)\right\}_{i=1}^{\infty}$ is the complete system of $W_{2}^{2}[0,1]$ and $\psi_{i}(x)=\left.L_{y} R_{x}(y)\right|_{y=x_{i}}$.

Proof. Note here that

$$
\begin{aligned}
\psi_{i}(x) & =\left(L^{*} \varphi_{i}\right)(x)=\left(\left(L^{*} \varphi_{i}\right)(y), R_{x}(y)\right) \\
& =\left(\varphi_{i}(y), L_{y} R_{x}(y)\right)=\left.L_{y} R_{x}(y)\right|_{y=x_{i}} .
\end{aligned}
$$

Clearly, $\psi_{i}(x) \in W_{2}^{2}[0,1]$.
For each fixed $u(x) \in W_{2}^{2}[0,1]$, let $\left(u(x), \psi_{i}(x)\right)=0, \quad(i=$ $1,2, \ldots)$, which means that

$$
\left(u(x),\left(L^{*} \varphi_{i}\right)(x)\right)=\left(L u(\cdot), \varphi_{i}(\cdot)\right)=(L u)\left(x_{i}\right)=0 .
$$

Since $\left\{x_{i}\right\}_{i=1}^{\infty}$ is dense on $[0,1],(L u)(x)=0$. It follows that $u \equiv 0$ from the existence of $L^{-1}$. So the proof of the Theorem 1 is complete.

Theorem 2. If $\left\{x_{i}\right\}_{i=1}^{\infty}$ is dense on $[0,1]$ and the solution of (6) is unique, then the solution of (6) is

$$
v(x)=\sum_{i=1}^{\infty} \sum_{k=1}^{i} \beta_{i k} h\left(x_{k}\right) \bar{\psi}_{i}(x) .
$$

Proof. Applying Theorem 1, it is easy to see that $\left\{\bar{\psi}_{i}(s)\right\}_{i=1}^{\infty}$ is the complete orthonormal basis of $W_{2}^{2}[0,1]$. 


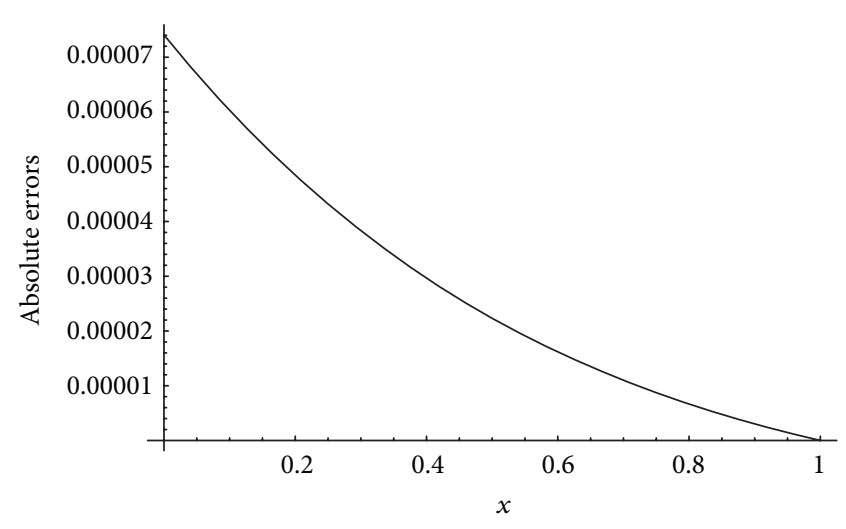

(a)

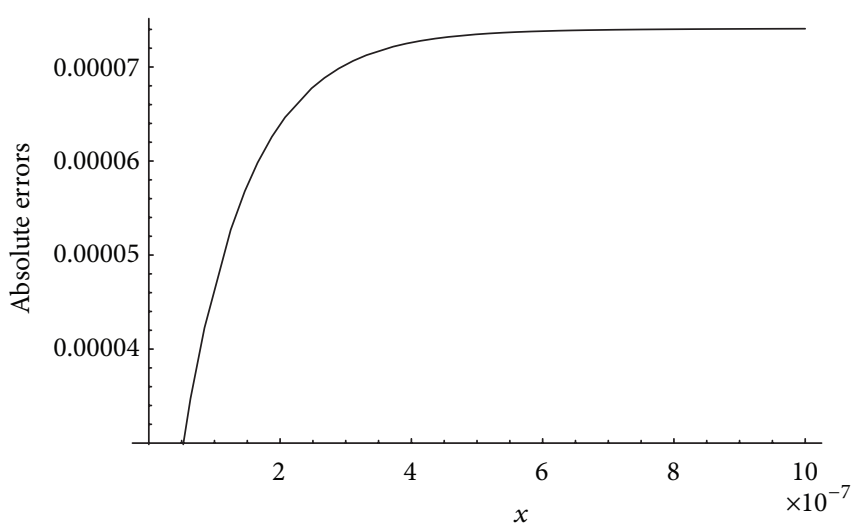

(b)

Figure 3: Absolute errors $\left|u(x)-u_{200}(x)\right|$ of Example 1 for $\varepsilon=10^{-7}$ ((a): regular domain; (b): boundary later domain).

TABLE 1: Comparison of maximum absolute error with other methods for $N=64$.

\begin{tabular}{lccc}
\hline$\varepsilon$ & {$[21]$} & {$[22]$} & Present method \\
\hline $2^{-12}$ & $1.49 e-002$ & $4.80 e-004$ & $2.60 e-005$ \\
$2^{-20}$ & $1.53 e-002$ & $1.87 e-006$ & $2.60 e-005$ \\
\hline
\end{tabular}

Note that $\left(w(s), \varphi_{i}(s)\right)=w\left(s_{i}\right)$ for each $w(x) \in W_{2}^{1}[0,1]$ hence we have

$$
\begin{aligned}
v(x) & =\sum_{i=1}^{\infty}\left(v(x), \bar{\psi}_{i}(x)\right) \bar{\psi}_{i}(x) \\
& =\sum_{i=1}^{\infty} \sum_{k=1}^{i} \beta_{i k}\left(v(x), L^{*} \varphi_{k}(x)\right) \bar{\psi}_{i}(x) \\
& =\sum_{i=1}^{\infty} \sum_{k=1}^{i} \beta_{i k}\left(L v(x), \varphi_{k}(x)\right) \bar{\psi}_{i}(x) \\
& =\sum_{i=1}^{\infty} \sum_{k=1}^{i} \beta_{i k}\left(h(x), \varphi_{k}(x)\right) \bar{\psi}_{i}(x) \\
& =\sum_{i=1}^{\infty} \sum_{k=1}^{i} \beta_{i k} h\left(x_{k}\right) \bar{\psi}_{i}(x)
\end{aligned}
$$

and the proof of the theorem is complete.

The approximate solution $v_{N}(x)$ can be obtained by taking finitely many terms in the series representation of $v(x)$ and

$$
v_{N}(x)=\sum_{i=1}^{N} \sum_{k=1}^{i} \beta_{i k} h\left(x_{k}\right) \bar{\psi}_{i}(x)
$$

\subsection{Solution of the Boundary Layer Domain. Consider}

$$
\begin{gathered}
\varepsilon u^{\prime \prime}(x)+a(x) u^{\prime}(x)+\sum_{i=1}^{m} b_{i}(x) u\left(q_{i} x\right) \\
=f(x), \quad 0<x<K \varepsilon, \\
u(0)=\alpha, \quad u(K \varepsilon)=\delta \triangleq u_{r, N}(K \varepsilon) .
\end{gathered}
$$

TABLE 2: Comparison of maximum absolute error with other methods for $N=256$.

\begin{tabular}{lccc}
\hline$\varepsilon$ & {$[21]$} & {$[22]$} & Present method \\
\hline $2^{-12}$ & $3.40 e-003$ & $4.86 e-004$ & $2.98 e-008$ \\
$2^{-20}$ & $3.90 e-002$ & $1.90 e-006$ & $2.98 e-008$ \\
\hline
\end{tabular}

For the boundary layer domain, we scale $x=\varepsilon s$ with $y(s) \equiv$ $u(x)$; then (15) becomes

$$
\begin{gathered}
y^{\prime \prime}(s)+a(\varepsilon s) y^{\prime}(y)+\varepsilon \sum_{i=1}^{m} b_{i}(\varepsilon s) y\left(q_{i} s\right) \\
=\varepsilon f(\varepsilon s), \quad 0<s<K, \\
y(0)=\alpha, \quad y(K)=\delta .
\end{gathered}
$$

Using the similar method for solving (3), we can obtain the approximate solution of (16) in the reproducing kernel space $W_{2}^{3}[0, K]=\left\{u(x) \mid u^{\prime \prime}(x)\right.$ is an absolutely continuous real valued function, $\left.u^{\prime \prime \prime}(x) \in L^{2}[0, K], u(0)=0, u(K)=0\right\}$, in which the inner product and norm are given, respectively, by

$$
\begin{gathered}
(u(y), v(y))_{W_{2}^{3}}=u(0) v(0)+u^{\prime}(0) v^{\prime}(0) \\
+u(K) v(K)+\int_{0}^{K} u^{\prime \prime \prime} v^{\prime \prime \prime} d y, \\
\|u\|_{W_{2}^{3}}=\sqrt{(u, u)_{W_{2}^{3}}} .
\end{gathered}
$$

The approximate solution of (16) can be represented by

$$
y_{N}(s)=\sum_{i=1}^{N} \sum_{k=1}^{i} \beta_{i k} g\left(s_{k}\right) \bar{\psi}_{i}(s),
$$

where $g(s)=\varepsilon f(\varepsilon s)$. Therefore, the approximation of solution $u_{l}(x)$ of boundary layer domain problem (15) can be obtained by

$$
u_{l, N}(x)=y_{N}\left(\frac{x}{\varepsilon}\right)
$$


TABLE 3: Comparison of computed solution with [22-25] for Example 4.2 with $N=100$ and $\varepsilon=10^{-3}$.

\begin{tabular}{lccccc}
\hline$x$ & Exact solution & Present method & {$[22]$} & {$[23]$} & -1.398575 \\
\hline 0.01 & -0.987875 & -0.987921 & -0.989854 & -1.603239 & -2.607798 \\
0.03 & -0.967160 & -0.967161 & -0.969099 & -1.579587 & -1.940047 \\
0.05 & -0.945600 & -0.945602 & -0.947500 & -1.550175 & -1.698810 \\
0.07 & -0.923240 & -0.923242 & -0.925100 & -1.519601 & -1.598463 \\
0.09 & -0.900080 & -0.900082 & -0.901900 & -1.503912 & -1.509999 \\
0.10 & -0.888200 & -0.888202 & -0.610000 & -0.657717 & -0.664829 \\
0.30 & -0.608600 & -0.608603 & -0.250000 & -0.074626 & -0.078895 \\
0.50 & -0.249000 & -0.249005 & 0.190000 & 0.615126 & 0.613702 \\
0.70 & 0.190600 & 0.190594 & 0.710000 & 1.000000 & 1.000000 \\
0.90 & 0.710200 & 0.710193 & 1.000000 & & \\
1.00 & 1.000000 & 1.000000 & &
\end{tabular}

From (5) and (19), the approximate solution of (1) on the entire region $[0,1]$ is immediately obtained as follows:

$$
u_{N}(x)= \begin{cases}u_{l, N}(x), & 0 \leq x \leq K \varepsilon \\ u_{r, N}(x), & K \varepsilon<x \leq 1\end{cases}
$$

\section{Numerical Examples}

Example 1. Consider the following singular perturbation problem with pantograph delay

$$
\begin{gathered}
\varepsilon u^{\prime \prime}(x)+e^{x} u^{\prime}(x)+\sin (x) u(x) \\
+u\left(\frac{x}{3}\right)+u\left(\frac{2 x}{3}\right)=f(x), \quad 0<x<1, \\
u(0)=3, \quad u(1)=3+\sinh (1),
\end{gathered}
$$

where $f(x)$ is given such that its exact solution is $u(x)=3+$ $\sinh (x)+2 x e^{-x / \varepsilon}$. Using the present method and taking $M=$ $1, K=10, x_{i}=i / N$ (regular domain), $s_{i}=K(i-1) /(N-$ 1) (boundary layer domain), $i=1,2, \ldots, N, N=200$ and $\varepsilon=10^{-3}, \varepsilon=10^{-5}, \varepsilon=10^{-7}$, respectively, the numerical results are given in Figures 1, 2, and 3. All computations are performed by using Mathematica 5.1.

Example 2. For comparison, we consider the following singular perturbation problem without delay [21-25]

$$
\begin{gathered}
\varepsilon u^{\prime \prime}(x)+u^{\prime}(x)=2 x+1, \quad 0<x<1, \\
u(0)=0, \quad u(1)=1 .
\end{gathered}
$$

Its exact solution is $u(x)=x(x+1-2 \varepsilon)+(2 \varepsilon-1)(1-$ $\left.e^{-x / \varepsilon}\right) /\left(1-e^{-1 / \varepsilon}\right)$. Using the present method and taking $M=1$, $K=10, x_{i}=i / N$ (regular domain), $s_{i}=K(i-1) /(N-1)$ (boundary layer domain), $i=1,2, \ldots, N$, the numerical results compared with other methods are given in Tables 1 , 2 , and 3 . It is shown that the present method can yield better results than existing methods.

\section{Conclusion}

In this paper, a new method is proposed for solving singularly perturbed multipantograph delay equations. The present method is based on the RKM, the asymptotic expansion technique, and the method of scaling. The major advantage of the method is that it can produce good globally continuous approximate solutions. The results from the numerical example show that the present method is an accurate and reliable analytical technique for treating singularly perturbed multipantograph delay equations.

\section{Conflict of Interests}

The authors declare that there is no conflict of interests regarding the publication of this paper.

\section{Acknowledgments}

The authors would like to express their thanks to the unknown referees for their careful reading and helpful comments. The work was supported by the NSFC (Grant nos. 11201041 and 11026200), the Special Funds of the National Natural Science Foundation of China (Grant no. 11141003), and Qing Lan Project of Jiangsu Province.

\section{References}

[1] G. M. Amiraliyev and F. Erdogan, "Uniform numerical method for singularly perturbed delay differential equations," Computers \& Mathematics with Applications, vol. 53, no. 8, pp. 1251-1259, 2007.

[2] I. G. Amiraliyeva, F. Erdogan, and G. M. Amiraliyev, "A uniform numerical method for dealing with a singularly perturbed delay initial value problem," Applied Mathematics Letters, vol. 23, no. 10, pp. 1221-1225, 2010.

[3] M. K. Kadalbajoo and K. K. Sharma, "Numerical analysis of singularly perturbed delay differential equations with layer behavior," Applied Mathematics and Computation, vol. 157, no. 1, pp. 11-28, 2004.

[4] M. K. Kadalbajoo and V. P. Ramesh, "Hybrid method for numerical solution of singularly perturbed delay differential equations," Applied Mathematics and Computation, vol. 187, no. 2, pp. 797-814, 2007. 
[5] M. K. Kadalbajoo and K. K. Sharma, "A numerical method based on finite difference for boundary value problems for singularly perturbed delay differential equations," Applied Mathematics and Computation, vol. 197, no. 2, pp. 692-707, 2008.

[6] M. K. Kadalbajoo and D. Kumar, "Fitted mesh B-spline collocation method for singularly perturbed differential-difference equations with small delay," Applied Mathematics and Computation, vol. 204, no. 1, pp. 90-98, 2008.

[7] G. M. Amiraliyev and E. Cimen, "Numerical method for a singularly perturbed convection-diffusion problem with delay," Applied Mathematics and Computation, vol. 216, no. 8, pp. 23512359, 2010.

[8] P. Rai and K. K. Sharma, "Numerical analysis of singularly perturbed delay differential turning point problem," Applied Mathematics and Computation, vol. 218, no. 7, pp. 3483-3498, 2011.

[9] M. Cui and Y. Lin, Nonlinear Numerical Analysis in the Reproducing Kernel Space, Nova Science, New York, NY, USA, 2009.

[10] A. Berlinet and C. Thomas-Agnan, Reproducing Kernel Hilbert Spaces in Probability and Statistics, Kluwer Academic, Boston, Mass, USA, 2004.

[11] F. Z. Geng and M. Cui, "Solving a nonlinear system of second order boundary value problems," Journal of Mathematical Analysis and Applications, vol. 327, no. 2, pp. 1167-1181, 2007.

[12] F. Z. Geng and M. Cui, "New method based on the HPM and RKHSM for solving forced Duffing equations with integral boundary conditions," Journal of Computational and Applied Mathematics, vol. 233, no. 2, pp. 165-172, 2009.

[13] F. Z. Geng, "A novel method for solving a class of singularly perturbed boundary value problems based on reproducing kernel method," Applied Mathematics and Computation, vol. 218, no. 8, pp. 4211-4215, 2011.

[14] F. Z. Geng, S. P. Qian, and S. Li, "A numerical method for singularly perturbed turning point problems with an interior layer," Journal of Computational and Applied Mathematics, vol. 255, pp. 97-105, 2014.

[15] B. Wu and X. Li, "Iterative reproducing kernel method for nonlinear oscillator with discontinuity," Applied Mathematics Letters, vol. 23, no. 10, pp. 1301-1304, 2010.

[16] W. Wang, B. Han, and M. Yamamoto, "Inverse heat problem of determining time-dependent source parameter in reproducing kernel space," Nonlinear Analysis: Real World Applications, vol. 14, no. 1, pp. 875-887, 2013.

[17] Y. Wang, M. Du, F. Tan, Z. Li, and T. Nie, "Using reproducing kernel for solving a class of fractional partial differential equation with non-classical conditions," Applied Mathematics and Computation, vol. 219, no. 11, pp. 5918-5925, 2013.

[18] M. Inc, A. Akgül, and A. Kiliçman, "A novel method for solving $\mathrm{KdV}$ equation based on reproducing kernel Hilbert space method," Abstract and Applied Analysis, vol. 2013, Article ID 578942, 11 pages, 2013.

[19] M. Mohammadi and R. Mokhtari, "Solving the generalized regularized long wave equation on the basis of a reproducing kernel space," Journal of Computational and Applied Mathematics, vol. 235, no. 14, pp. 4003-4014, 2011.

[20] G. Akram and H. U. Rehman, "Numerical solution of eighth order boundary value problems in reproducing kernel space," Numerical Algorithms, vol. 62, no. 3, pp. 527-540, 2013.

[21] M. K. Kadalbajoo and P. Arora, "B-spline collocation method for the singular-perturbation problem using artificial viscosity," Computers \& Mathematics with Applications, vol. 57, no. 4, pp. 650-663, 2009.
[22] A. Kaushik, V. Kumar, and A. K. Vashishth, "An efficient mixed asymptotic-numerical scheme for singularly perturbed convection diffusion problems," Applied Mathematics and Computation, vol. 218, no. 17, pp. 8645-8658, 2012.

[23] A. Andargie and Y. N. Reddy, "Fitted fourth-order tridiagonal finite difference method for singular perturbation problems," Applied Mathematics and Computation, vol. 192, no. 1, pp. 90$100,2007$.

[24] M. M. Chawla, "A fourth-order tridiagonal finite difference method for general non-linear two-point boundary value problems with mixed boundary conditions," IMA Journal of Applied Mathematics, vol. 21, no. 1, pp. 83-93, 1978.

[25] M. M. Chawla and P. N. Shivakumar, "An efficient finite difference method for two-point boundary value problems," Neural, Parallel \& Scientific Computations, vol. 4, no. 3, pp. 387395, 1996. 


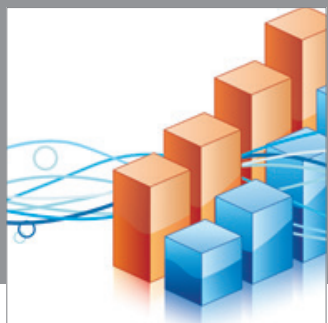

Advances in

Operations Research

mansans

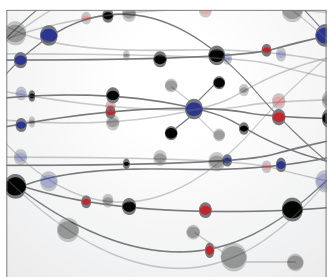

The Scientific World Journal
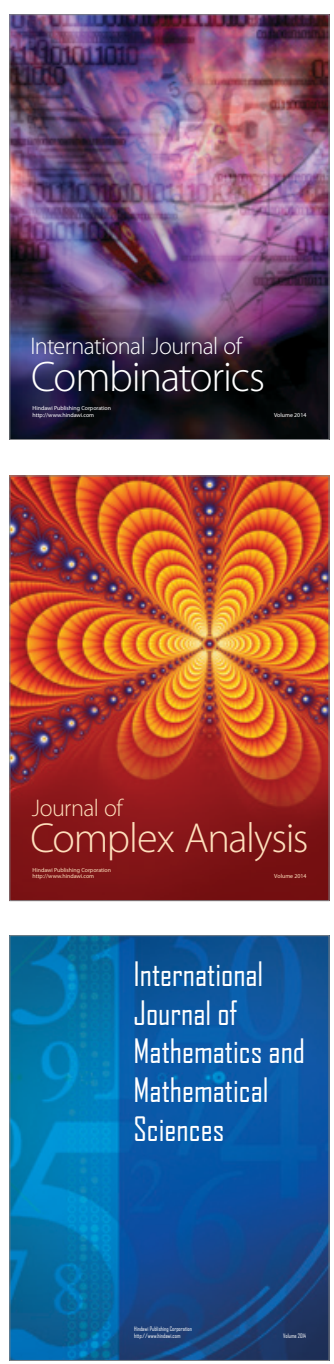
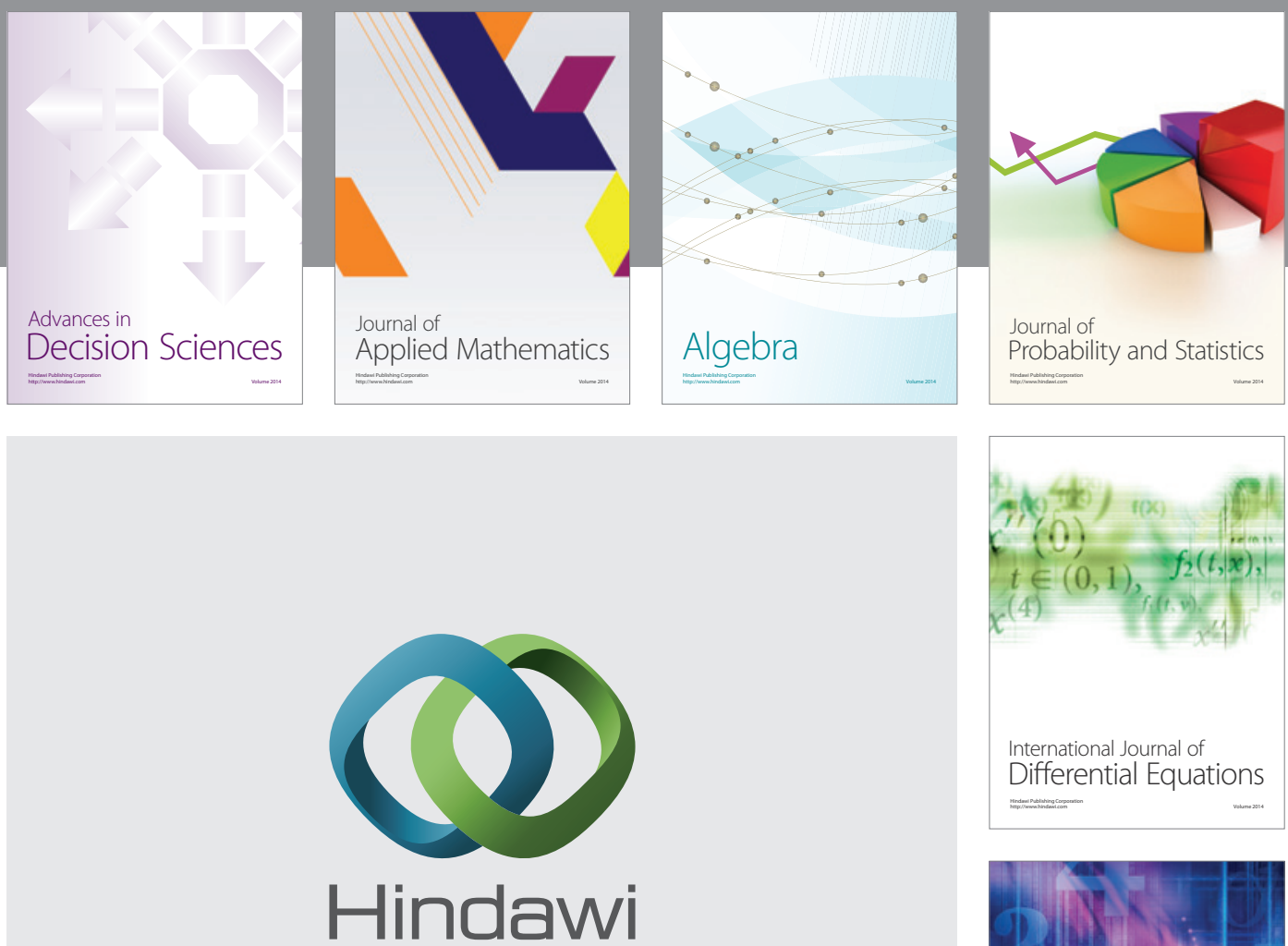

Submit your manuscripts at http://www.hindawi.com
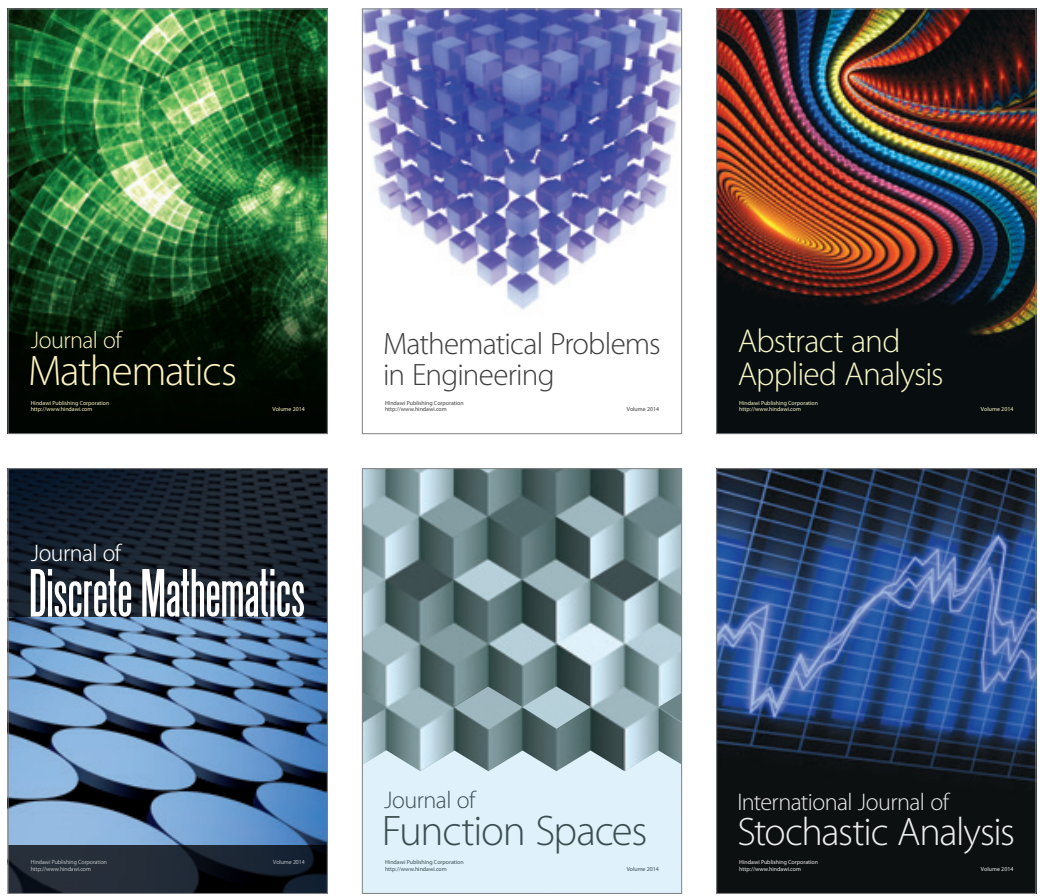

Journal of

Function Spaces

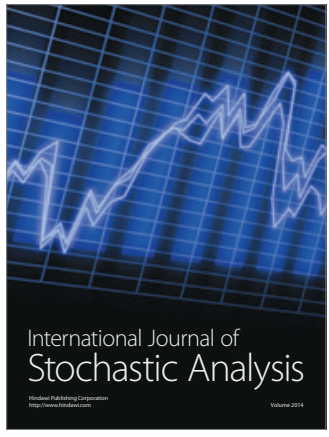

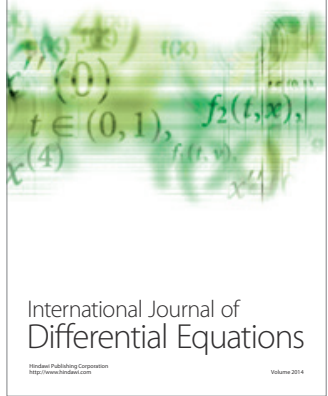
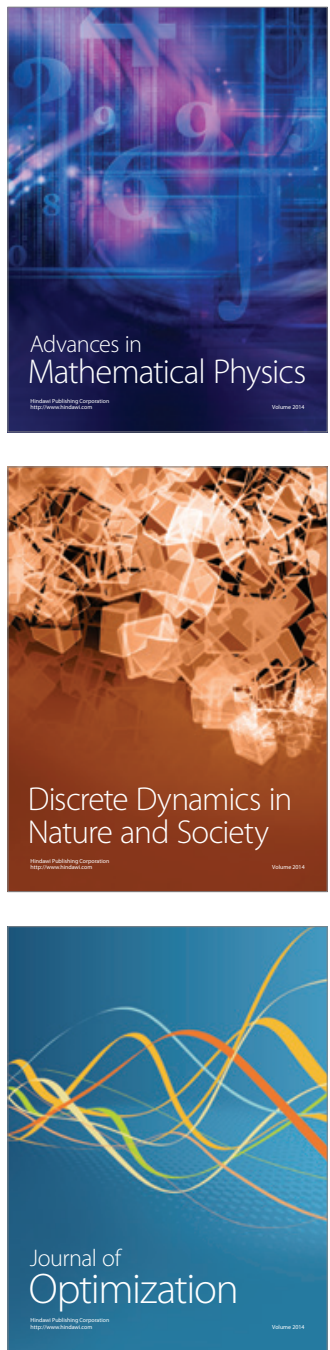